\section{Condições de implantação e operação da farmacovigilância na indústria farmacêutica no Estado de São Paulo, Brasil}

\author{
Pharmacovigilance implementation and operational \\ conditions in the pharmaceutical industry in \\ São Paulo State, Brazil
}

\author{
${ }^{1}$ Faculdade de Ciências \\ Farmacêuticas, Universidade \\ de São Paulo, São Paulo, \\ Brasil. \\ 2 Faculdade de Saúde \\ Pública, Universidade \\ de São Paulo, \\ São Paulo, Brasil. \\ Correspondência \\ N.S. Romano-Lieber \\ Departamento de Prática \\ de Saúde Pública, Faculdade \\ de Saúde Pública. \\ Universidade de São Paulo. \\ Av. Dr. Arnaldo 715 \\ São Paulo, SP \\ 01246-904, Brasil. \\ nicolina@usp.br
}

\section{Abstract}

The development of new drugs and limitations of clinical trials have increased the likelihood of adverse drug events. Pharmacovigilance is essential to detect and evaluate adverse drug events, thereby reducing risks and avoiding excessive public health costs. This study focused on the pharmacovigilance programs in the pharmaceutical industry in São Paulo State, Brazil. Data were collected through questionnaires sent electronically to 105 companies, $41.9 \%$ of which responded. The main reason for implementing pharmacovigilance programs was to comply with legal requirements, while the main justification for its absence was production limited to herbal remedies, officinal products, and supplements. The article discusses the obstacles to program implementation, resources used, and characteristics of several such programs. Conclusions: (a) standardization is the reason for the increasing number of programs and reports, but more specific guidelines are needed; (b) results depend on multi-sector involvement; (c) customer service centers are an important source of reports; and (d) operation of the service requires only modest human and material resources.

Drug Evaluation; Controlled Clinical Trials; Drug Industry
Márcia Sayuri Takamatsu Freitas 1 Nicolina Silvana Romano-Lieber 2

\section{Introdução}

A Organização Mundial da Saúde (OMS) 1 (p. 7) define a farmacovigilância como "a ciência e as atividades relacionadas à detecção, avaliação, compreensão e prevenção dos efeitos adversos ou qualquer outro possivel problema relacionado a medicamentos". Seu alcance inclui fitoterápicos, medicamentos tradicionais e complementares, produtos derivados de sangue, produtos biológicos, aparelhos médicos e vacinas 1 .

Um dos objetivos da farmacovigilância é a detecção de eventos não previstos ou não observados até a fase III do estudo clínico dos medicamentos, anterior à sua comercialização. Trata-se de ensaios realizados em condições rigorosas, em que há ausência de pacientes com contraindicações potenciais (gestantes, idosos, crianças) ou que façam uso de outros tratamentos e que são bastante restritos quanto ao número de pacientes e tempo de duração, diferentemente do que ocorre na prática clínica habitual. Além disso, são também funções da farmacovigilância: (1) a detecção de reações adversas a medicamentos previstas na literatura, com a finalidade de estimar a sua real incidência na população; (2) a detecção de interações medicamentosas; (3) a identificação e quantificação dos fatores de riscos relativos ao uso dos medicamentos e (4) a análise e disseminação das informações obtidas entre os profissionais de saúde e para a população 2 . 
Dentre os eventos adversos a medicamentos, suas reações são de particular interesse para a indústria farmacêutica, pois, conforme a OMS 3 , são efeitos prejudiciais ou indesejáveis que se apresentam após a administração das doses normalmente utilizadas no homem para a profilaxia, o diagnóstico e o tratamento de uma enfermidade. As reações adversas a medicamentos também representam um problema de saúde pública pois, ainda que, em geral, possam ser de caráter leve ou moderado, também podem levar à morte ou ser responsáveis por lesões irreversíveis 4 .

Lawson 5 relata que 3 a $6 \%$ das admissões hospitalares ocorrem devido a reações adversas a medicamentos e que, entre os pacientes hospitalizados, 10 a $20 \%$ sofreriam alguma reação adversa a medicamentos. Em determinadas situações, as reações adversas a medicamentos podem levar à morte até $1 \%$ dos pacientes ${ }^{6}$. Para Vidotti 7 , prevenir a ocorrência de reações adversas a medicamentos tem implicações, tanto na qualidade de vida do paciente quanto no sistema de saúde, o qual gastaria menos com os tratamentos recorrentes advindos da ocorrência das reações adversas a medicamentos.

Em países da Europa como Inglaterra e França, bem como nos Estados Unidos e Japão, as indústrias farmacêuticas, já há vários anos, devem relatar compulsoriamente às autoridades sanitárias, informações de segurança sobre seus produtos, tanto aquelas geradas em ensaios clínicos quanto as relativas a produtos já disponíveis no mercado ${ }^{8}$.

No Brasil, ainda não existe normatização federal a esse respeito. Entretanto, desde 1998, foi implantado no Estado de São Paulo um sistema que permite o controle do uso dos medicamentos e o conhecimento dos seus efeitos sobre a saúde da população, denominado Programa Estadual de Redução de Iatrogenias, sob a coordenação do Centro de Vigilância Sanitária do Estado de São Paulo (CVS-SP) 9. A partir de 2000, com a publicação da Portaria CVS 10 10, as empresas detentoras de registro de medicamentos devem encaminhar ao CVS-SP as notificações espontâneas de eventos adversos ocorridos com o uso de medicamentos, relatados por profissionais de saúde, possibilitando uma experiência local para este procedimento.

O objetivo deste trabalho foi conhecer os programas de farmacovigilância de indústrias farmacêuticas sediadas no Estado de São Paulo, bem como avaliar o impacto da publicação da Portaria CVS 10 na implantação daqueles programas. Também se buscou conhecer os programas de farmacovigilância em relação aos seus recursos humanos e materiais, além das diferentes dificuldades observadas na sua implantação.

\section{Material e métodos}

A pesquisa foi realizada em empresas farmacêuticas sediadas no Estado de São Paulo. Para seleção das empresas a serem pesquisadas partiu-se de uma lista extra-oficial de empresas registradas na Agência Nacional de Vigilância Sanitária (ANVISA) acrescida de uma relação de empresas fornecida pelo Sindicato das Indústrias Farmacêuticas do Estado de São Paulo (SINDUSFARMA) e por empresas constantes do Dicionário de Especialidades Farmacêuticas (DEF) 11 como sediadas no Estado. Dessa relação de 682 empresas, foram excluídas as empresas fabricantes de medicamentos veterinários, insumos, correlatos, cosméticos, produtos de higiene e embalagens. Restaram 302 empresas, fabricantes de medicamentos alopáticos, homeopáticos, fitoterápicos, produtos farmacopeicos, dietéticos, imunobiológicos e hemoderivados. Para validação da relação de empresas a serem pesquisadas, consultou-se o sítio da ANVISA na Internet (http:// www.anvisa.gov.br). As empresas foram classificadas quanto ao número de registros de que dispunham naquela instituição. Determinaram-se como pequenas aquelas empresas com menos de 50 registros, como médias, aquelas com 50 a 100 registros e grandes as que tinham mais de 100 registros. As empresas que não constavam do sítio ou que não apresentavam registro de produtos foram excluídas da análise. Desta forma, restaram 171 empresas a serem pesquisadas. Utilizou-se como critério para o envio da pesquisa a existência de endereço eletrônico para contato com a área de registro ou de farmacovigilância das empresas, obtido em consulta ao SINDUSFARMA. Assim, foram encaminhados questionários a 105 empresas. Esta fração é equivalente a $61,4 \%$ das empresas farmacêuticas sediadas no Estado de São Paulo, com produto registrado na ANVISA.

O período da pesquisa foi do início de janeiro a 31 de março de 2003.

Utilizou-se para a coleta de dados um questionário com questões abertas e fechadas, o qual foi pré-testado em três indústrias farmacêuticas. O questionário foi dividido em três partes. A primeira parte do instrumento foi dedicada à identificação da empresa, sua origem e à caracterização da presença ou ausência de um programa de farmacovigilância. Em caso de vigência de um programa, passava-se à segunda parte, dedicada à coleta de informações relativas ao mesmo, como o tempo de implantação, dificuldades, além dos recursos humanos e materiais envolvidos. A terceira parte destinou-se ao conhecimento dos resultados do programa, buscando-se informações referentes ao recebimento das noti- 
ficações e ao tratamento dado a elas. Assim, foram pesquisadas as principais fontes de notificação, fluxo da informação na empresa e a existência de avaliação de associação entre o medicamento e as reações notificadas, aqui chamada "causalidade".

Os questionários foram enviados pela Internet. Para atender à questão ética, foi enviado também um termo de responsabilidade, no qual os pesquisadores comprometiam-se a não divulgar o nome das empresas colaboradoras e a utilizar as informações recebidas apenas para fins acadêmicos.

\section{Resultados}

Quarenta e quatro empresas (41,9\%) daquelas selecionadas retornaram os questionários. Destas, $20(45,5 \%)$ possuíam programa de farmacovigilância; 12 (27,3\%) não possuíam o programa, mas $6(13,6 \%)$ delas responderam ao questionário; 4 $(9,1 \%)$ informaram que estavam implantando o programa, mas não responderam às questões e $8(18,2 \%)$ informaram que não poderiam colaborar com a pesquisa. Desta forma, foram 26 os questionários respondidos e utilizados na análise, correspondendo a $24,7 \%$ do total de instrumentos enviados.

A Tabela 1 apresenta a situação das empresas que atenderam à pesquisa, em relação à presença de programa de farmacovigilância e segundo o número de registros na ANVISA.

Quanto à caracterização das empresas que responderam ao questionário, 14 eram de capital multinacional, 11 nacionais, uma joint venture $\mathrm{e}$ as demais não foi possível caracterizar. Das vinte empresas que apresentavam o programa, 13 eram multinacionais e sete nacionais.

Em nove (45\%) empresas o programa funcionava há aproximadamente dois anos. Os programas restantes funcionavam por períodos que variavam de 1 a 20 anos.

Na Tabela 2 são apresentadas as razões citadas por 11 empresas para implantação do programa de farmacovigilância. As justificativas apresenta-

Tabela 1

Empresas farmacêuticas sediadas no Estado de São Paulo, Brasil, que foram pesquisadas quanto à presença de programa de farmacovigilância segundo o número de registros na Agência Nacional de Vigilância Sanitária (ANVISA).

\begin{tabular}{|c|c|c|c|c|c|c|c|c|c|c|c|c|}
\hline \multirow{3}{*}{$\begin{array}{l}\text { Número de } \\
\text { produtos }\end{array}$} & \multicolumn{6}{|c|}{ Programa de farmacovigilância } & \multicolumn{2}{|c|}{ Não colaborou } & \multicolumn{2}{|c|}{ Não retornou } & \multicolumn{2}{|c|}{ Total } \\
\hline & \multicolumn{2}{|c|}{ Sim } & \multicolumn{2}{|c|}{ Não } & \multicolumn{2}{|c|}{ Implantando } & \multirow[b]{2}{*}{$n$} & \multirow[b]{2}{*}{$\%$} & \multirow[b]{2}{*}{$n$} & \multirow[b]{2}{*}{$\%$} & \multirow[b]{2}{*}{$n$} & \multirow[b]{2}{*}{$\%$} \\
\hline & $n$ & $\%$ & $\mathrm{n}$ & $\%$ & $n$ & $\%$ & & & & & & \\
\hline$<50$ & 8 & 14,8 & 7 & 12,9 & 4 & 7,4 & 5 & 9,3 & 30 & 55,6 & 54 & 100,0 \\
\hline $50-100$ & 5 & 16,7 & 3 & 10,0 & 0 & 0,0 & 3 & 10,0 & 19 & 63,3 & 30 & 100,0 \\
\hline$>100$ & 7 & 43,8 & 2 & 12,5 & 0 & 0,0 & 0 & 0,0 & 7 & 43,8 & 16 & 100,0 \\
\hline Sem informação & 0 & 0,0 & 0 & 0,0 & 0 & 0,0 & 0 & 0,0 & 5 & 100,0 & 5 & 100,0 \\
\hline Total & 20 & 19,0 & 12 & 11,4 & 4 & 3,8 & 8 & 7,6 & 61 & 58,1 & 105 & 100,0 \\
\hline
\end{tabular}

Tabela 2

Razões apontadas por 11 empresas farmacêuticas do Estado de São Paulo, Brasil, para implantação de um programa de farmacovigilância.

\begin{tabular}{|c|c|c|}
\hline Razões para implantação de um programa de farmacovigilância & n & $\%$ \\
\hline Atender exigências regulatórias nacionais & 7 & 63,6 \\
\hline Atender exigências regulatórias internacionais & 4 & 36,4 \\
\hline Uniformizar-se com a política interna internacional (matriz) para segurança do produto & 4 & 36,4 \\
\hline Ampliar o conhecimento sobre o perfil de segurança dos produtos em comercialização & 4 & 36,4 \\
\hline Ter responsabilidade ética, confirmando o compromisso com pacientes e profissionais de saúde & 2 & 18,2 \\
\hline Identificação, avaliação e prevenção dos efeitos adversos e outros problemas relacionados aos seus produtos & 1 & 9,1 \\
\hline Vontade da própria empresa/Implantação espontânea & 1 & 9,1 \\
\hline
\end{tabular}


das para a não implantação de um programa de farmacovigilância são apresentadas na Tabela 3.

As dificuldades relatadas pelas indústrias farmacêuticas para implantação de um programa de farmacovigilância foram classificadas como humanas internas, humanas externas e materiais e estão apresentadas na Tabela 4 .

Em 11 (55\%) das empresas a farmacovigilância estava subordinada à área médica, por necessitar do profissional médico para avaliação da suspeita de reação adversa. Em duas (10\%) empresas a farmacovigilância estava associada à área de registro/assuntos regulatórios. Nas outras sete (35\%) empresas observou-se uma diversidade de áreas envolvidas com a farmacovigilância: Garantia da Qualidade, Boas Práticas de Fabricação, Técnico-Científico, Pesquisa Clínica e Atendimento ao Consumidor.

Com relação aos recursos humanos envolvidos, observou-se que em 14 (70\%) empresas havia médico. Havia um número ainda maior de farmacêuticos, além de biomédicos e biólogos. Também foi apontada a atuação de estagiários, alunos de cursos de farmácia e biologia. A estrutura mais comum contava com no mínimo dois funcionários e no máximo sete, sendo mais freqüente a estrutura com três funcionários, observada em cinco (25\%) empresas. Entretanto, um programa relatou ter 16 profissionais envolvidos. Neste caso, em particular, havia a participação inclusive de um químico e de uma nutricionista.

Em 16 empresas (80\%) os funcionários recebiam treinamento e em duas (10\%) não. Em oito das multinacionais o treinamento do responsável pela área era realizado na matriz ou por meio de Procedimento Operacional Padrão (POP). As informações eram depois disseminadas entre os responsáveis pelos setores envolvidos, em geral, as áreas de Serviço de Atendimento ao Consumidor (SAC) e Vendas/Representantes, os quais treinavam seus funcionários. Este treinamento das empresas multinacionais é anual ou adotado quando há alteração na legislação vigente. As empresas nacionais adotam variados tipos de atualização como autotreinamento, cursos externos ou materiais preparados pela própria empresa.

Justificativas apresentadas por nove empresas farmacêuticas do Estado de São Paulo, Brasil, para a não implantação de um programa de farmacovigilância.

\begin{tabular}{|c|c|c|}
\hline Justificativas para a não implantação de um programa de farmacovigilância & $\mathbf{n}$ & $\%$ \\
\hline Produzir fitoterápicos, produtos oficinais e suplementos alimentares e não produtos farmacêuticos propriamente ditos & 4 & 44,4 \\
\hline Possuir Serviço de Atendimento ao Cliente, o qual se responsabiliza por encaminhar as reclamações e solucioná-las & 3 & 33,3 \\
\hline Receber pequeno número de suspeitas de reações adversas & 1 & 11,1 \\
\hline Licencia os produtos para outras empresas as quais se responsabilizam pelo programa & 1 & 11,1 \\
\hline
\end{tabular}

Tabela 4

Dificuldades relatadas por nove indústrias farmacêuticas do Estado de São Paulo, Brasil, para implementação de programa de farmacovigilância.

\begin{tabular}{|c|c|}
\hline Tipo de dificuldade & Descrição \\
\hline Humanas internas & $\begin{array}{l}\text { Falta de conhecimento por parte dos funcionários; implantação de nova cultura de farmacovigilância; } \\
\text { resistência por parte do setor de Marketing/Vendas }\end{array}$ \\
\hline Humanas externas & $\begin{array}{l}\text { Recusa e/ou falta de hábito dos profissionais de saúde em relatar efeitos adversos; dificuldade no } \\
\text { retorno das informações por parte dos médicos }\end{array}$ \\
\hline \multirow[t]{5}{*}{ Materiais } & Indefinição da Agência Nacional de Vigilância Sanitária (ANVISA) quanto à farmacovigilância nas \\
\hline & indústrias; ausência de legislação nacional regulamentando a farmacovigilância; ausência de dicionário \\
\hline & para codificação dos eventos; diferenças entre legislação internacional e nacional; adequação de \\
\hline & formulários com informações necessárias; variedade de formulários utilizados; falta de sistemas \\
\hline & (softwares) adequados \\
\hline
\end{tabular}


Quanto aos recursos materiais, 100\% das empresas contavam com linha telefônica e computador para armazenar dados, 95\% possuíam aparelho de fax e acesso à rede de computadores, $90 \%$ tinham impressora e $80 \%$ dispunham de fotocopiadora.

Os materiais de consulta citados foram: livros (Martindale e PDR), dicionários (Dicionário de Especialidades Farmacêuticas, Dorlands, Medical Dictionary for Regulatory Activities - MedDRA - e Stedman) e bancos de dados eletrônicos (BIREME, MEDLINE, Council for International Organizations of Medical Sciences - CIOMS - e Micromedex). Também foram citados periódicos como Periodic Safety Update Reports (PSUR) e outros relacionados.

O principal meio de captação de relatos de suspeita de reações adversas apontado foi o SAC citado por 13 (65\%) das empresas que contavam com o programa. Algumas empresas contavam também com notificações provenientes das áreas de Pesquisa Clínica e Força de Vendas/Representantes. Houve ainda quem relatasse a literatura científica como fonte de notificação.

O fluxo do tratamento das notificações mostrou-se bastante semelhante: em 11 (55\%) das empresas as notificações eram repassadas ao setor de farmacovigilância para avaliação de pertinência. Quando se tratava de um evento adverso, as informações eram transcritas em formulário específico, armazenadas em bancos de dados ou enviadas para o CVS-SP, ANVISA e matriz, quando coubessem, dentro dos prazos estipulados. No caso de multinacionais, as matrizes encaminhavam o relato ao Centro Internacional de Farmacovigilância (WHO Collaborating Centre for International Drug Monitoring), em Uppsala, Suécia.

Quanto ao formulário para recebimento das notificações, o modelo do CIOMS foi adotado em $60 \%$ dos casos. Outros modelos citados foram o da ANVISA, o da FDA Safety Information and Adverse Event Reporting Program (MEDWATCH; http://www.fda.gov/medwatch/) e o da CVS-SP (http://www.cvs.saude.sp.gov.br), disponíveis nos respectivos sítios na Internet. Quatro (30,8\%) empresas entre as multinacionais adotavam o modelo próprio de suas matrizes.

Oito (40\%) empresas realizavam avaliação de causalidade, dez (50\%) não o faziam, sendo que seis (30\%) repassavam às suas matrizes todos os relatos sem avaliação prévia. As demais não responderam à pergunta. Os instrumentos citados para a avaliação foram: algoritmo de Naranjo/Kramer 12, algoritmo de Karch \& Lasagna 13 e duas empresas utilizavam algoritmos elaborados por suas matrizes. Quatro (50\%) das oito empresas não responderam qual o instrumento empregado.
Quatorze (70\%) empresas responderam sobre os tipos de relatos recebidos, "eventos adversos esperados" ou "eventos adversos não esperados", sendo estes últimos quaisquer eventos não mencionados na bula atual do medicamento 10 . A proporção de relatos de "eventos esperados" esteve entre 3,98 e $100 \%$, sendo que em seis (43\%) empresas essa proporção esteve entre 92 e $99 \%$. Com relação aos "eventos não esperados", em 11 (78,6\%) empresas a proporção esteve entre 0,99 e $60 \%$ e, em três $(21,4 \%)$ empresas, esteve em torno de $5 \%$. Também se questionaram a proporção de "queixas técnicas sobre o medicamento", ou seja, problemas técnicos do produto, relacionados com sua qualidade, desempenho ou segurança 14 e a proporção de "reações adversas a medicamento". Com relação às queixas técnicas/reações adversas a medicamentos, dez (50\%) empresas responderam. A proporção relatada foi de 3 a 100\%, sendo que em três (30\%) esteve entre 10 e $20 \%$.

Onze empresas (55\%) divulgaram seu programa de farmacovigilância aos prescritores por meio de equipe de vendas ou SAC. Em cinco empresas $(45,1 \%)$ que fizeram divulgação, verificou-se aumento na quantidade de notificações recebidas. O impacto também foi observado na melhoria da qualidade das mesmas. Para alguns, não foi observada alteração, por tratar-se de programa recente ou por ser inexpressivo o número de relatos anterior à divulgação do programa.

\section{Discussão}

O número de empresas que respondeu ao questionário permite fazer uma descrição dos programas de farmacovigilância existentes na indústria farmacêutica paulista à época da pesquisa. A garantia do anonimato dos respondentes bem como o fato de algumas empresas terem respondido à pesquisa apenas para comunicar que não responderiam ao questionário permite supor que as respostas obtidas aproximam-se da realidade dos programas pesquisados.

Dentre as empresas farmacêuticas que responderam ao questionário e que fazem farmacovigilância de seus produtos, o maior número é de multinacionais. Estas também apresentam programas com maior tempo de duração que os relativos às empresas de capital nacional, devido à determinação de suas matrizes, em cujos países de origem o programa é obrigatório.

Os resultados sugerem que a ausência de um programa de farmacovigilância parece estar ligada ao número de produtos registrados pela empresa, conforme mostra a Tabela 1. Assim, quanto menor o número de produtos, menor seria o interesse ou a necessidade de se implantar um 
programa de farmacovigilância. Observa-se, na mesma tabela, que a porcentagem dos não respondentes não difere entre as classes estabelecidas na população examinada, permitindo supor que os resultados obtidos entre os respondentes representam uma boa amostragem da situação, que não depende do tamanho da empresa em relação ao número de produtos.

Parece ter havido impacto da Portaria CVS 10, em relação à implantação de programa de farmacovigilância nas indústrias farmacêuticas, uma vez que a maior parte das empresas implantou o programa após a sua publicação. Isto também pode ser observado na Tabela 2 , na qual observase que a principal razão citada para implantação de um programa de farmacovigilância é a exigência da legislação.

Talbot \& Nilsson ${ }^{8}$, ao traçar perspectivas para a farmacovigilância na indústria farmacêutica, já reconheciam a importância ao atendimento dos aspectos regulatórios formais mas salientavam que essa atividade deveria estar mais centrada na ciência, na busca de instrumentos que permitissem avaliar o impacto dos tratamentos para a saúde pública além de seus custos. O mesmo foi observado por Santini 15 e Edwards 16. Nessa convergência, algumas empresas relataram possuir um programa de farmacovigilância pelo seu compromisso com o usuário, atestando a segurança do produto comercializado.

Na Tabela 3, observa-se que empresas produtoras de fitoterápicos, suplementos alimentares e produtos oficinais se abstiveram de implantar programas de monitorização de eventos adversos, alegando que seus produtos não necessitam passar pelo processo de vigilância pós-comercialização. Esta idéia não encontra respaldo na legislação brasileira. Além disso, a OMS preconiza a observação dos efeitos causados por esses medicamentos 2 . Neste particular, Barnes 17 apontou a necessidade de desenvolver práticas diferenciadas de farmacovigilância para fitoterápicos, a fim de aumentar o número de relatos de seus eventos adversos. Observou que esses produtos apresentam problemas relativos a sua denominação, obtenção e utilização, uma vez que os usuários, em geral, não buscam orientação profissional para o uso e nem relatam efeitos adversos.

Também o pequeno número de suspeitas de reação adversa foi utilizado como justificativa para a ausência de farmacovigilância. Entretanto, deve-se ressaltar que os relatos de eventos adversos, ainda que não graves, são importantes para quantificar a sua real incidência e para a descrição das características desses eventos, além de servirem como subsídio ao prescritor na avaliação do risco/benefício do uso daquele medicamento para determinado paciente.
As principais dificuldades citadas para a implantação do programa estão relacionadas às dificuldades para obtenção de relatos de reações adversas a medicamentos. Alguns fatores relacionados à não notificação de reação adversa a medicamento pelos médicos são listados por Polimeni et al. 18. Por exemplo, a dificuldade em reconhecer que o quadro clínico que se apresenta possa ser uma reação adversa ao medicamento prescrito; o fato de prescritores acreditarem no sistema de aprovação e regulamentação de medicamentos como sendo seguros; sentimentos de culpa por acreditarem que o tratamento proposto causou a reação adversa a medicamento; medo de parecerem ignorantes quanto às reações já conhecidas; falta de tempo e dificuldade para interpretação dos termos usados em farmacovigilância. Por estes motivos, o sucesso do programa depende, também, da divulgação do programa junto aos notificadores.

Burt 19 apresenta três sugestões para o aumento na quantidade e na qualidade de relatos de eventos adversos, incluindo a necessidade de uma central para onde os dados possam ser encaminhados e avaliados rapidamente, proeminência de farmacologia clínica no currículo dos cursos de medicina e o encorajamento dos prescritores a preencher relatos completos. Não é sem razão, portanto, que foi observado um aumento na quantidade e qualidade de relatos de suspeitas de eventos adversos por aquelas empresas que divulgaram seu programa junto aos médicos.

Houve também resistência de setores da empresa sobre os benefícios da notificação de eventos adversos, especialmente os setores de Marketing/Vendas por não visualizarem a farmacovigilância como fator positivo para a imagem do produto. Ao contrário, a atenção da empresa quanto aos efeitos de seus produtos pode aumentar a confiança do prescritor e do usuário. Os resultados sugerem que parece haver mais sucesso no programa quando há sensibilização de várias áreas para a necessidade de conhecer os eventos adversos causados pelos produtos da empresa. Isto foi observado por Sayers 20 que, ao descrever o sucesso de um programa de farmacovigilância, ressaltou a importância da comunicação do conceito de qualidade a todo grupo.

Outras dificuldades apontadas para a implantação parecem, atualmente, mais fáceis de solucionar, pois se referem aos recursos materiais como a padronização dos formulários e programa de computadores acessíveis para o armazenamento dos dados. Esta limitação, já apontada pela indústria farmacêutica em outros países, resultou no desenvolvimento de um dicionário (MedDRA) criado para padronizar a terminolo- 
gia médica utilizada para fins regulatórios. Este instrumento facilita o diálogo da indústria com as autoridades regulatórias, além de facilitar a análise e transmissão de dados entre diferentes países, conforme descrito por Goldman 21.

Quanto aos recursos humanos envolvidos, verifica-se que a atividade na empresa é multiprofissional, sendo fundamental a presença de médicos e farmacêuticos para superar eventuais dificuldades, relativas à disciplinaridade, na busca de informações complementares junto aos notificadores. De qualquer forma, a educação continuada no tema parece ser fator importante para a manutenção da qualidade do programa.

A análise dos resultados permite afirmar que, embora haja muitas diferenças entre os programas apresentados, é possível atuar com uma estrutura mínima de recursos humanos. Esta estrutura mínima seria viabilizada pela presença do SAC, o qual estaria encarregado da captação de relatos, enquanto os profissionais da farmacovigilância poderiam concentrar-se na busca e processamento das informações. Lewis 22 descreve uma experiência em que o uso de um banco de dados mundial, aliado a um fluxo de procedimentos adequado na empresa, permite, a um número relativamente pequeno de funcionários, agilizar a análise de relatos de casos ao mesmo tempo em que realizam outras funções requeridas pela farmacovigilância. Quanto aos recursos materiais citados, estes são materiais de referência de fácil acesso.

A principal fonte de captação de suspeita de eventos adversos foi o SAC. Este serviço tornou-se obrigatório a partir de 8 de outubro de 1998, com a publicação da Portaria $n$. 802 da ANVISA 23. Com isto, aumentou a possibilidade de prescritores e usuários relatarem eventos adversos. É possível, ainda, supor que com a obrigatoriedade desse serviço haja um aumento do número de notificações, graças à pressão da legislação vigente e de um maior questionamento por parte dos usuários. Além disso, a recente estruturação do programa de farmacovigilância do Estado de São Paulo e da Unidade de Farmacovigilância da ANVISA facilita ao notificador o encaminhamento do relato.

Nem todas as empresas multinacionais fazem, no Brasil, uma avaliação prévia, por meio de algoritmo, da relação de causalidade entre o evento adverso e o medicamento utilizado. Provavelmente, isto se deve à obrigatoriedade de relatar todas as notificações recebidas, independentemente de relação causal, como preconizado, por exemplo, na legislação americana. Em algumas empresas nacionais, os dados obtidos na notificação são encaminhados para o CVS-SP, sem nenhuma avaliação.
Diante da possibilidade da publicação de normatização relativa à farmacovigilância na indústria farmacêutica brasileira 24,25, acredita-se que algumas das dificuldades apontadas para a implantação dos programas sejam minimizadas. Desta forma, o estabelecimento de formulários padronizados, da atuação de prescritores e usuários, bem como da determinação de quais produtos estão sujeitos a monitoramento contribuirão para um incremento no número de programas e, conseqüentemente, da qualidade e quantidade das informações.

Foi constatado, entre as empresas pesquisadas e junto ao CVS-SP, na época da pesquisa que, no âmbito do Estado de São Paulo, a indústria farmacêutica tem colaborado com a instituição na proposição de um formulário de notificações de fácil preenchimento pelos notificadores, visando um aumento no número de notificações. Esta instituição, desde o início de suas atividades de farmacovigilância, já possuía um banco de dados único para armazenar dados de notificação de hospitais, profissionais de saúde e indústria farmacêutica. Atualmente, estão sendo implantados sistemas diferenciados para armazenar dados de notificação das indústrias farmacêuticas. A partir da conclusão deste banco será possível analisar com mais facilidade os dados coletados.

O objeto da farmacovigilância deve ser a segurança do usuário do medicamento. Entretanto, a indústria farmacêutica também tem vantagens quando faz o acompanhamento dos seus produtos. De acordo com Falci 26, há vantagens do ponto de vista ético, ao assegurar que os riscos associados com o uso dos produtos sejam bem entendidos pelo médico e pelo usuário; legal, ao assegurar o atendimento adequado às exigências de legislação sanitária; científico, ao promover um melhor conhecimento biológico quanto ao uso do produto no ser humano; e comercial, ao proporcionar o melhor conhecimento da relação risco/benefício do produto, facilitando a análise mercadológica. A consecução desses propósitos depende da implantação e operação eficaz desses serviços no âmbito das empresas. Neste sentido, este trabalho permite concluir:

(a) A promoção de normas e regulamentos pelo setor público é uma força eficiente de motivação. Todavia a falta de esclarecimentos mais específicos às partes envolvidas tem permitido interpretações dúbias, como no caso dos fitoterápicos;

(b) O padrão de resultados depende em grande parte do número de setores envolvidos tanto na empresa como fora dela. Este trabalho mostra que o sucesso da iniciativa pode ser obtido a partir da promoção interna de cursos e palestras de atualizações. Com isto, a farmacovigilância se 
destaca como um elo importante de integração entre os diversos setores da empresa;

(c) Ao contrário do que possa ser alegado, a estrutura interna de operação de tal serviço pode ser relativamente pouco dispendiosa em termos de recursos humanos e materiais;

(d) O SAC foi a maior fonte de captação de relatos de eventos adversos;

(e) O fato de o CVS-SP exigir das empresas apenas os dados de notificações realizadas por pro-

\section{Resumo}

O desenvolvimento de novos produtos farmacêuticos e as limitações dos ensaios clínicos controlados têm sido responsáveis pelo aumento das possibilidades de eventos adversos a medicamentos. A farmacovigilância é essencial para detectar e avaliar eventos adversos a medicamentos reduzindo riscos e evitando gastos excessivos em saúde pública. Este trabalho objetivou conhecer programas de farmacovigilância de indústrias farmacêuticas sediadas no Estado de São Paulo, Brasil. Os dados foram coletados utilizando-se questionário enviado eletronicamente a 105 empresas, das quais $41,9 \%$ retornaram. O motivo principal para implantação de programas foi atender exigências regulatórias e a principal justificativa para sua ausência foi produção limitada aos produtos fitoterápicos, oficinais e suplementos. Discutem-se as dificuldades para implantação dos programas, os recursos utilizados e características de alguns deles. Conclusões: (a) a normatização é fonte de motivação para o aumento dos programas e do número de relatos mas é insuficiente sem esclarecimentos mais específicos; (b) os resultados dependem de envolvimento multisetorial; (c) o Serviço de Atendimento ao Consumidor é importante fonte de relatos; (d) a estrutura da operação do serviço pode ser pouco dispendiosa em termos de recursos humanos e materiais.

Avaliação de Medicamentos; Ensaios Clínicos Controlados; Indústria Farmacêutica fissionais de saúde pode fazer com que aquelas que não possuem o programa de farmacovigilância não relatem as notificações recebidas de usuários (por exemplo, via SAC), se isentando da obrigatoriedade do envio de relatório de notificações e da busca mais aprofundada de dados, omitindo informações que poderiam contribuir com o programa mundial.

\section{Colaboradores}

M. S. T. Freitas participo da revisão bibliográfica, do trabalho de campo (envio dos questionários) e da redação do artigo. N. S. Romano-Lieber participou da revisão bibliográfica e da redação do artigo. 


\section{Referências}

1. WHO Collaborating Center for International Drug Monitoring. The importance of pharmacovigilance. Safety monitoring of medicinal products. Uppsala: WHO Collaborating Center for International Drug Monitoring; 2002.

2. Figueras A, Napchan BM, Mendes GB. Farmacovigilância - ação na reação. São Paulo: Centro de Vigilância Sanitária; 2002.

3. World Health Organization. International drug monitoring: the role of national centers. Geneva: World Health Organization; 1972. (Technical Report Series, 498).

4. Tognoni G, Laporte JR. Estudos de utilização de medicamentos e de farmacovigilância. In: Laporte JR, Tognoni G, Rozenfeld S, organizadores. Epidemiologia do medicamento: princípios gerais. São Paulo: Editora Hucitec/Rio de Janeiro: ABRASCO; 1989. p. 43-56.

5. Lawson DH. Epidemiology. In: Davies DM, editor. Textbook of adverse drug reactions. $4^{\text {th }}$ Ed. Oxford: Oxford University Press; 1991. p. 5-17.

6. Porter J, Jick, H. Drug related deaths among medical inpatients. JAMA 1977; 237:879-81.

7. Vidotti CCF. A tênue fronteira entre a cura e o malefício. Pharmacia Brasileira 2000; 22:36-9.

8. Talbot JC, Nilsson BS. Pharmacovigilance in the pharmaceutical industry. Br J Clin Pharmacol 1998; 45:427-31.

9. Resolução n. 72, de 13 de abril de 1998. Institui o Programa Estadual de Redução das Iatrogenias. Diário Oficial do Estado de São Paulo 1998; 14 abr.

10. Portaria CVS 10, 22 de novembro de 2000. Dispõe sobre o fluxo da ficha de notificação de Eventos adversos e dá outras providências. Diário Oficial do Estado de São Paulo 2000; 22 nov.

11. Dicionário de especialidades farmacêuticas - DEF 2002/03. 31a Ed. Rio de Janeiro: Editora de Publicações Científicas; 2002.

12. Naranjo CA, Busto U, Seliers EM, Sandor P, Ruiz I, Roberts EA, et al. A method for estimating the probability of adverse drug reactions. Clin Pharmacol Ther 1981; 30:239-45.

13. Karch FE, Lasagna L. Evaluation adverse drug reactions. Adverse Drug React Bull 1976; 59:204-9.

14. Moretto LD. A era da farmacovigilância. Pharmaceutical Technology 2000; 4:43-8.

15. Santini C. La responsabilite de l'industrie pharmaceutique en matiere de securite sanitaire. Ann Pharm Fr 1999; 57:10-5.
16. Edwards IR. The accelerating need for pharmacovigilance. J R Coll Physicians Lond 2000; 34:48-51.

17. Barnes J. Pharmacovigilance of herbal medicines: a UK perspective. Drug Saf 2003; 26:829-51.

18. Polimeni G, Roncoroni S, Russo A, Cupani C, Iacobelli M, Caputi AP. Il ruolo di un sito internet nell'educazione medica continua in farmacovigilanza. In: 30o Congresso Nazionale della Società Italiana di Farmacologia. http://www.farmacovigilanza.org.poster/genova (acessado em 09/ Fev/2004).

19. Burt RA. Pharmacovigilance: three sugestions for improving the quantity of adverse event reports. Drug Inf J 2000; 34:229-38.

20. Sayers J. Implementing a quality management system in a global pharmacovigilance processing center. Quality Assurance Journal 2001; 5:71-5.

21. Goldman SA. Adverse event reporting and standardized medical terminologies: strengths and limitation. Drug Inf J 2002; 36:439-44.

22. Lewis DJ. Pharmacovigilance within the European Union: process improvement within a company safety unit. Pharm Med 1995: 9:225-38.

23. Portaria n. 802, de 8 de outubro de 1998. A Secretaria de Vigilância Sanitária do Ministério da Saúde institui o Sistema de Controle e Fiscalização em toda cadeia dos produtos farmacêuticos. Diário Oficial da União 1999; 4 fev.

24. Consulta Pública n. 5, de 14 de janeiro de 2002. A Agência Nacional de Vigilância Sanitária adota a publicação da Consulta Pública para que sejam apresentadas críticas e sugestões relativas à proposta de Regulamentação Técnica que dispõe sobre a notificação de eventos adversos sérios de produtos sujeitos à vigilância sanitária. Diário Oficial da União 2002; 15 jan.

25. Consulta Pública n. 10, de 9 de abril de 2003. A Agência Nacional de Vigilância Sanitária adota a publicação da Consulta Pública para que sejam apresentadas críticas e sugestões relativas à proposta de Resolução que estabelece atribuições ao Centro de Nacional Monitorização de Medicamentos. Diário Oficial da União 2003; 11 abr.

26. Falci M. A informação de medicamentos na indústria farmacêutica. In: Bonfim JRA, Mercucci VL, organizadores. A construção da política de medicamentos. São Paulo: Editora Hucitec/Sociedade Brasileira de Vigilância de Medicamentos; 1997. p. 175-7.

Recebido em 10/Mar/2005

Versão final reapresentada em 10/Abr/2006

Aprovado em 25/Abr/2006 